\title{
Formulation of Nanostructured lipid particles
}

\author{
Kavita Rani ${ }^{1}$, Amit Kumar J. Raval ${ }^{2}$, Dinesh Kaushik ${ }^{3}$, Rajesh Khathuriya ${ }^{4}$ \\ ${ }^{1}$ Ph. D Scholar, Pacific Academy of Higher Education And Research University Udaipur,Rajasthan,India \\ ${ }^{2}$ Supervisor, Associate Professor, Pacific Academy of Higher Education And Research University Udaipur, \\ Rajasthan, India \\ ${ }^{3}$ Co-Supervisor, Associate Professor, Hindu College of Pharmacy, Sonipat, Haryana, India \\ ${ }^{4}$ Associate Profeesor, Pacific College of Pharmacy, (Pacific Academy of Higher Education And Research \\ University Udaipur), Udaipur, Rajasthan, India
}

\section{*Correspondence}

Kavita Rani

Ph. D Scholar, Pacific Academy of Higher Education and Research University Udaipur, Rajasthan, India.

E-mail: kavitasharma.pharma@gmail.com

Received: 04-02-2020 / Revised: 20-06-2020 / Accepted: 26-07-2020

\begin{abstract}
Lipid nanocarriers are developed as an alternative to polymeric nanoparticles, liposomes and emulsions. NLCs are the second generation lipid carriers developed to overcome problems associated with Solid Lipid Nanoparticles and are utilized in various therapeutic approaches. NLCs were used for the delivery of lipophilic drugs .Biocompatible nature of lipids is responsible for its development as a good drug delivery. It was found to be having excellent characteristics over other lipid formulations.
\end{abstract}

Keywords:Lipid, carriers, NLCs.

This is an Open Access article that uses a fund-ing model which does not charge readers or their institutions for access and distributed under the terms of the Creative Commons Attribution License (http://creativecommons.org/licenses/by/4.0) and the Budapest Open Access Initiative (http://www.budapestopenaccessinitiative.org/read), which permit unrestricted use, distribution, and reproduction in any medium, provided the original work is properly credited.

\section{Introduction}

Nanostructured lipid carriers (NLC) are a delivery • system in which partial-crystallized lipid particles with $\bullet$ mean radii $\leq 100 \mathrm{~nm}$ are dispersed in an aqueous phase $\bullet$ containing emulsifier(s), as a potential delivery system • may have some advantages in certain circumstances when compared with other colloidal carriers[1]. NLC have been developed to overwhelm the drawbacks affiliated with SLN. They are advised to be the second lifetime of lipid nanoparticles. Contrasted to SLN, NLC show a higher loading capability for hardworking compounds by conceiving a less organized solid lipid matrix, i.e. by blending a fluid lipid with the solid lipid, a higher element drug stacking can be achieved. Thus, the NLC have an expanded drug stacking capacity in evaluation to SLN and the likelihood of drug expulsion during storage is less[2]
Advantages of nanostructured lipid carriers over other lipid-based formulation

Higher drug loading and entrapment potential.

Modulation of drug release pattern.

Long-term stability of incorporated drug during storage

Minimum level of surfactant with maximum drug loading potential[3].

Limitations

- Cytotoxic effects related to the nature of matrix and concentration,

- Irritative and sensitising action of some surfactants,

- Application and efficiency in case of protein and peptide drugs and gene delivery systems still need to be better exploited, and

- Lack of sufficient preclinical and clinical studies with these nanoparticles in case of bone repair[4]. 


\section{Structure of NLC's}

The structure of NLS's are very and somehow similar to SLNs, NLCs have three very specific features. Hese properties are based up on the location the drug is going to be integrated three different methods were adopted for a development and formulation of nanostructure NLCs.

- NLC type I also called as imperfect crystal.

- NLC type II also called as multiple type.

- NLC type III also called as amorphous type

Methods of preparation of NLS's

Here are several methods are developed for the preparation of NLS's

- High-pressure homogenization.

- Microemulsion technique.

- Evaporation solvent injection.

- Multiple emulsion techniques.

- Phase inversion.

- Ultrasonication.

- Membrane contractor technique

High pressure homogenization

High Pressure Homogenization Technique has been used as a reliable and powerful technique for the largescale production of NLCs. It involves the melting of solid lipid materials first before mixing them with liquid lipid and drugs. After mixing, the molten liquid is scattered throughout the aqueous phase, which contains surfactants. The mixture is stirred to form the beginning of an emulsion. Lipid emulsions are pushed with high pressure (100-200bars) through a narrow gap of few micron ranges. So shear stress and cavitations are the forces which cause the disruption of particle to submicron range. Normally the lipid contents are in the range of $5-10 \%$. In contrast to other preparation technique, high pressure homogenization does not show scaling up problem. Homogenization may be performed either at elevated temperature (hot homogenization) or below room temperature (cold homogenization) [5]

\section{Hot high pressure homogenization}

Hot homogenization is carried out at temperatures above the melting point of the lipid $\left(100^{\circ} \mathrm{C}\right.$ above the melting point of the lipid) and can therefore be regarded as the homogenization of an emulsion. A preemulsion of the drug loaded lipid melt and the aqueous emulsifier phase (same temperature) is obtained by a high shear mixing device (Ultra-Turrax). The quality of the pre emulsion affects the quality of the final product to a large extent, and obtaining droplets in the size range of a few micrometers is desirable. In general, higher temperatures result in lower particle sizes because of the decreased viscosity of the inner phase. Hot homogenization has three basic problems. The first is temperature dependent degradation of the drug, the second is the drug penetrates into the aqueous phase during homogenization and the third is complexity of the crystallization step of the nanoemulsion leading to several modifications and/or supercooled melts [6]

Cold high pressure homogenization

Cold homogenization has been developed to overcome the problems of the hot homogenization technique such as, temperature mediated accelerated degradation of the drug payload, partitioning and hence loss of drug into the aqueous phase during homogenization. This method is suitable for heat-labile drugs or hydrophilic drugs. the cold homogenization is carried out with the solid lipid without melting as done in hot process. Drug along with lipid in solid state is milled to form microparticles, and further dispersed in a solution containing emulsifier. The pre-suspension formed is then subjected to high pressure homogenization at or below room temperature.In the cold HPH technique, lipid is melted above its melting point and drug is dissolved or dispersed in it. The system is cooled down by means of dry ice or liquid nitrogen. After solidification, the lipid mass is grounded using ball or mortar milling to yield lipid microparticles in a range between 50 and $100 \mu \mathrm{m}$. Then a microemulsion is formed by adding these microparticles into cold surfactant solution with stirring. This suspension is passed through a high pressure homogenizer at/or below room temperature and the microparticles are broken down to nanoparticles[7].

\section{Microemulsion technique}

The lipids (fatty acids or glycosides eg. lipid acid) are liquified and in this liquefied lipid the drug is dissolved. A mixture of water, surfactant and cosurfactant is heated at the same temperature as the lipid and added to the lipid melt under mild stirring. A clean microemulsion was obtained when the components were mixed in correct ratio. The formed microemulsion is the basis for the nanoparticle formation of a requisite size. This microemulsion is then dispersed in a cold aqueous medium under mild mechanical mixing of hot microemulsion with water during a quantitative relation in the range 1:25-1:50. This dispersion in cold aqueous medium leads to rapid recrystalization of the oil droplets. Surfactant and co-surfactant include lecithin, salt along with alcohol such as Butanol. The microemulsion was prepared in a large temperaturecontrolled tank and then pumped from this tank into a cold water tank for precipitation purpose[8].

\section{Solvent emulsification-evaporation technique}

This method is used for the production of the polymeric nanoparticles by solvent evaporation in $\mathrm{o} / \mathrm{w}$ emulsion via precipitation. In this technique the lipophillic material and hydrophobic drug were 
dissolved in a water immiscible organic solvent (eg. Cyclohexane, toluene, chloroform) and then that is emulsified in an aqueous phase using high speed homogenization. The lipid precipitates upon evaporation of solvent and thus forming the nanoparticles. The big advantage of this technique is avoidance of any thermal stress, which makes it appropriate for incorporation of highly thermo labile drugs. A clean disadvantage is the use of organic solvent which may interact with drug molecule and limited the solubility of the lipid in the organic solvent [9].

\section{Solvent emulsification-diffusion technique}

It consists of two different phases; organic phase and aqueous phase. In this technique organic solvent used (eg. Benzyl alcohol, ethyl acetate, methyl acetate, isopropyl acetate) must be partially miscible in water. Initially both the phases were mutually saturated in order to ensure the initial thermodynamic equilibrium of both the liquids. When heating is required for the solubilization of the liquid the saturation step was performed at elevated temperature. Then the drug and the lipid were dissolved in aqueous phase and the organic phase was emulsified with aqueous solution containing stabilizer using mechanical stirrer. After formation of this $\mathrm{o} / \mathrm{w}$ emulsion water (dilution medium) in typical ratio ranges from 1:5 to $1: 10$, were added to the system in order to allow solvent diffusion to the continuous phase, forming the nanoparticles. In the whole process continuous stirring was maintained. The obtained dispersion can be placed in vacuum desiccators for 24 hours to evaporate the residual organic solvent

\section{Melting dispersion method}

In this method, drug, solid lipid and organic solvents were melted together and the aqueous phase (water phase) is heated at the same temperature as organic phase separately. The organic phase is then added to the water phase with high speed stirring for few hours. The resulting mixture is cooled down to room temperature to obtain nanoparticles[10,11].

High-Pressure
Homogenization (HPH)
•lipid is melted at $5-10^{\circ} \mathrm{C}$ above
its melting point and mixed with
the drug. (a)
• hot aqueous surfactant solution
was added (pre-emulsion) (b)
-the pre-emulsion was put into
the high-pressure homogenizer.
•cooled down to room
temperature

\begin{tabular}{|c|}
\hline Solvent Diffusion \\
•Similar to "solvent \\
Emulsification-Evaporation" but in \\
this case, lipid is dissolved in a \\
partially water-miscible organic \\
solvents. \\
•the solvent diffuse to the liquid \\
phase and the lipid solidified. \\
\hline
\end{tabular}

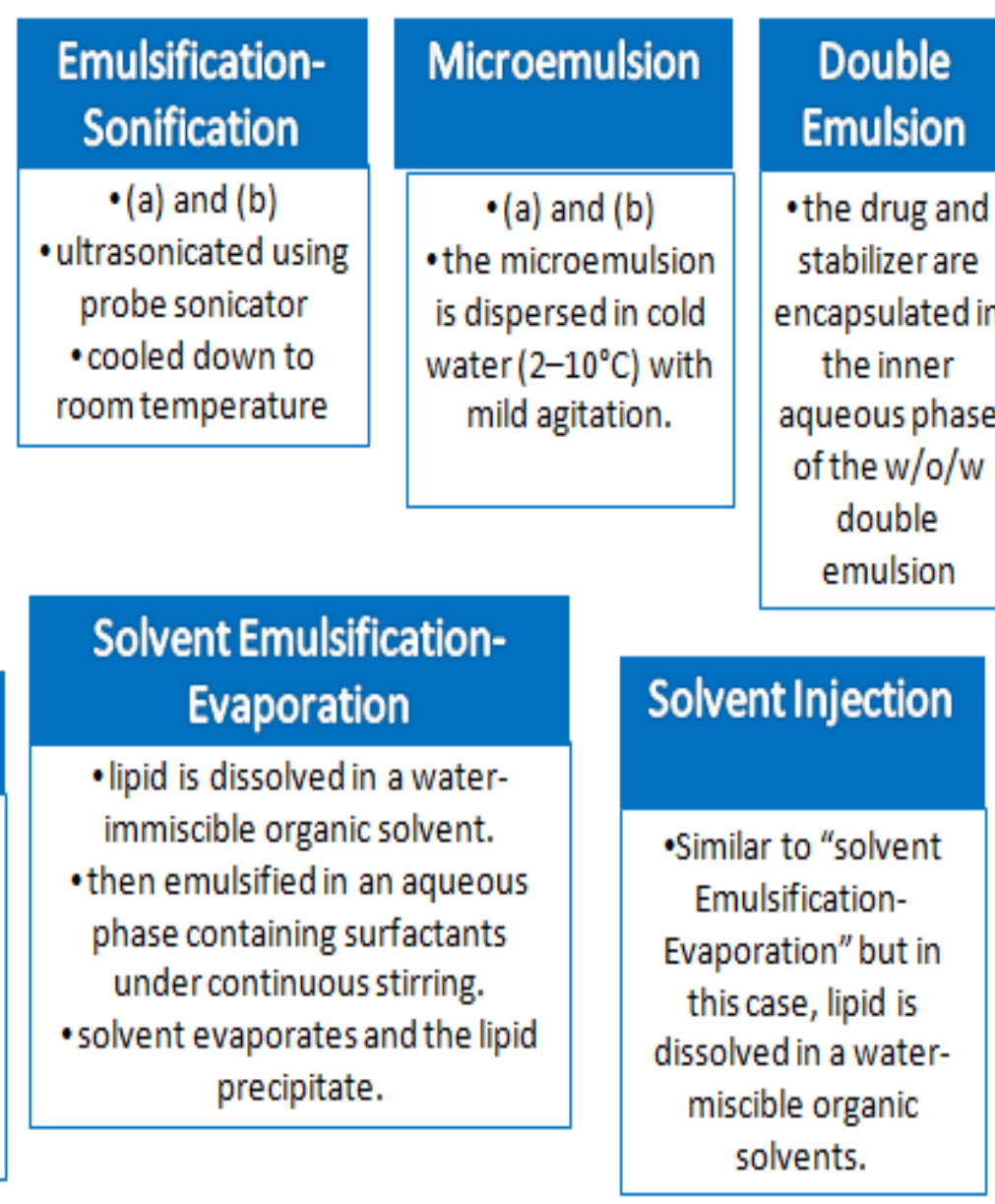

Fig 1: NLC Method of preparation 


\section{Material and methods}

Tetanus toxoid was obtained from Panacea Biotec, New Delhi, India.All the materials used were of analytical grade.

\section{Methods}

Nanostructured lipid particles (NLPs) of stearic acid were used as an immune-adjuvant for delivery of tetanus toxoid (TT) antigen. Further, cationic lipid (DDAB18) was incorporated in the composition of NLPs to induce the cationic charge . Cationic nanoparticles have the ability to improve the transport of an antigen in to the dendritic cells and consequently the humoral and cellular immunity. Hence, TT was adsorbed on to the surface of non-aggregated NLPs (TT-NLPs1) and non-aggregated CNLPs (TT-C-NLPs 1-10) by adsorption method that were constructed by solvent diffusion method.[12-15].

\section{Preparation and optimisation of cationic nanostructured lipid particles}

Nanostructured lipid particles (NLPs) and cationic nanostructured lipid particles (C-NLPs) were prepared by solvent diffusion method[16,17]. In brief, 50mg of stearic acid was dissolved in a mixture of $6 \mathrm{~mL}$ ethanol and $6 \mathrm{~mL}$ acetone to prepare the organic phase. Subsequently, this organic phase was dispersed in 120 $\mathrm{mL}$ of distilled water that was maintained at $70{ }^{0} \mathrm{C}$. Both phases were cooling to room temperature, water in the mixture was evaporated stirred for $30 \mathrm{~min}$ whilst the temperature was kept constant[18-21]. Upon cooling to room temperature, water in the mixture was evaporated by using a lyophilizer to get the fine powder of NLPs. Correspondingly C-NLPs were prepared by altering and optimising the composition of lipid matrix. The stearic acid (50 mg) was mixed with a gradient concentration of DDMB18 (50-250 mg) in the equal volume of organic phase and processed under identical conditions as recommended in NLPs. Nanoparticles were sterilised by autoclave at $121{ }^{\circ} \mathrm{C}$ for for $15 \mathrm{~min}$ before loading the antigen.

Table 1: Optimisation and characterisation of tetanus toxoid-loaded nanostructured lipid particles

\begin{tabular}{|l|l|}
\hline Compositions & Stearic acid: DDAB18 \\
\hline TT-NLPs1 & $50 \mathrm{mg}: 0 \mathrm{mg}$ \\
\hline TT-C-NLPs1 & $50 \mathrm{mg}: 50 \mathrm{mg}$ \\
\hline TT-C-NLPs2 & $50 \mathrm{mg}: 100 \mathrm{mg}$ \\
\hline TT-C-NLPs3 & $50 \mathrm{mg}: 150 \mathrm{mg}$ \\
\hline TT-C-NLPs4 & $50 \mathrm{mg}: 200 \mathrm{mg}$ \\
\hline TT-C-NLPs5 & $50 \mathrm{mg}: 250 \mathrm{mg}$ \\
\hline TT-C-NLPs6 & $50 \mathrm{mg}: 300 \mathrm{mg}$ \\
\hline TT-C-NLPs7 & $50 \mathrm{mg} ; 350 \mathrm{mg}$ \\
\hline TT-C-NLPs8 & $50 \mathrm{mg}: 400 \mathrm{mg}$ \\
\hline TT-C-NLPs9 & $50 \mathrm{mg}: 450 \mathrm{mg}$ \\
\hline TT-C-NLPs10 & $50 \mathrm{mg}: 500 \mathrm{mg}$ \\
\hline
\end{tabular}

TT-NLPs: Tetanus toxoid-loaded nanostructured lipid particles;

TT-C-NLPs: Tetanus toxoid loaded cationic nanostructure lipid particles;

DDAB18: Dimethyl dioctadecylammonium bromide

The formulations were prepared and were subjected to different evaluation parameters.

\section{Conclusions}

NLC is a potential approach for improving the bioavailability of highly lipophilic drugs with poor aqueous solubility, extensive first pass metabolism, affinity for P-gp efflux transporters, and susceptibility to intra-enterocyte metabolism. In a way, the high caliber of NLC system shadows the petition of other lipid-based dosage form. Disrupted matrices of NLC consequently lead to higher drug loading, higher drug entrapment, modulated drug release and ultimately enhanced drug absorption as compared with other lipid-based formulations (SLN and LE) having uniform matrices of lipids. These special features of NLC are exclusively attributed to their unique composition, which is constituted of a blend of incompatible solid and liquid lipids. New nanomaterials are still being created, new effects discovered and new models and concepts developed to explain and understand the observed experimental results. The high potential of nanoparticles for applications has moved them from basic research, solely driven by scientific curiosity, into the focus of technological interest 


\section{References}

1. Domb AJ. 1995. Long acting injectable oxytetracycline-liposphere formulations. Int J Pharm. 124:271-278.

2. Domingo C, Saurina J. 2012. An overview of the analytical characterization of nanostructured drug delivery systems: towards green and sustainable pharmaceuticals: a review. Anal Chim Acta. 744:8-22.

3. Eldem T, Speiser P, Hincal A. 1991. Optimiza tion of spray-dried and congelated lipid micropellets and characterization of their surface morphology by scanning electron microscopy. Pharm Res. 8:47-54

4. Esposito E, Mariani P, Ravani L, Contado C, Volta M, Bido S, et al. 2012. Nanoparticulate lipid dispersions for bromocriptine delivery: characterization and in vivo study. Eur $\mathbf{J}$ Pharm Biopharm. 80:306-314.

5. Matsumura Y, Maeda H: A new concept for macromolecular therapeutics in cancer chemotherapy: mechanism of tumoritropic accumulation of proteins and the antitumor agent. Cancer Res. 1968, 6: 193-210.

6. Mehnert W, Mader K: Solid lipid nanoparticles: production, characterization and applications. Adv Drug Deliv Rev. 2001, 47: 165-196.

7. Muller RH, Mader K, Gohla S: Solid lipid nanoparticles (SLN) for controlled drug delivery - a review of the state of the art. Eur J Pharm Biopharm. 2000, 50: 61-177.

8. Pratt WB, Ruddon RW, Ensminger WD, Maybaum J: The Anticancer Drugs. 1994, New York: Oxford University Pres

9. Cavalli R, Caputo O, Gasco MR: Solid lipospheres of doxorubicin and idarubicin. Int J Pharm. 1993, 89: 9-12.

10. Zur Muhlen A, Schwarz C, Mehnert W: Solid lipid nanoparticles (SLN) for controlled drug delivery - drug release and release mechanism. Eur J Pharm Biopharm. 1998, 45: 149-155.

11. Cavalli R, Caputo O, Gasco MR: Preparation and characterization of solid lipid nanospheres containing paclitaxel. Eur J Pharm Sci. 2000,
10: 305-309. 10.1016/S0928-0987(00)00081-

6

12. Hahn SM, Russo A, Cook JA, Mitchell JB: A multidrug-resistant breast cancer line induced by weekly exposure to doxorubicin. Int $\mathrm{J}$ Oncol. 1999, 14: 273-279

13. Campone M, Vavasseur F, Le MT, Cabellec $\mathrm{K}$, Meflah FM, Vallette L: Induction of chemoresistance in HL-60 cells concomitantly causes a resistance to apoptosis and the synthesis of P-glycoprotein. Leukemia. 2001, 15: 1377-1387. 10.1038/sj.leu.2402222

14. Moghimi SM, Szebeni J: Stealth liposomes and long circulating nanoparticles: critical issues in pharmacokinetics, opsonization and protein-binding properties. Prog Lipid Res. 2003, 42: 463-478.

15. Park JW: Liposome-based drug delivery in breast cancer treatment. Breast Cancer Res. 2002, 4: 95-99. 10.1186/bcr432

16. Zara GP, Cavalli R, Bargoni A, Fundaro A, Vighetto D, Gasco MR: Intravenous administration to rabbits of non-stealth and stealth doxorubicin-loaded solid lipid nanoparticles at increasing concentrations of stealth agent: pharmacokinetics and distribution of doxorubicin in brain and other tissues. J Drug Target. 2002, 10: 327-335.

17. Barratt GM: Therapeutic applications of colloidal drug carriers. Pharm Sci Technolo Today. 2000, 3: 163-171. 10.1016/S14615347(00)00255-8

18. Mehnert $\mathrm{W}$, Mader $\mathrm{K}$ : Solid lipid nanoparticles: production, characterization and applications. Adv Drug Deliv Rev. 2001, 47: 165-196. 10.1016/S0169-409X(01)00105-

19. Mainardes RM, Silva LP: Drug delivery systems: past, present, and future. Curr Drug Targets. 2004, 5: 449-455

20. Alexis F, Rhee JW, Richie JP, RadovicMoreno AF, Langer R, Farokhzad OC. 2008. New frontiers in nanotechnology for cancer treatment. Urol Oncol. 26:74-85.

21. Araújo J, Gonzalez E, Egea MA, Garcia ML, Souto EB. 2009. Nanomedicines for ocular NSAIDs: safety on drug delivery. Nanomedicine. 5:394-401

\section{Source of Support: Nil \\ Conflict of Interest: Nil}

Kavita et al ASIAN PACIFIC JOURNAL OF NURSING AND HEALTH SCIENCES, 2020; 3(2):21-24

